\title{
Quantum Hall droplets in coupled lateral quantum dots
}

\author{
Ramin M. Abolfath, Pawel Hawrylak, Michel Pioro-Ladriere, and Andy Sachrajda \\ Institute for Microstructural Sciences, National Research Council of Canada, Ottawa, K1A OR6, Canada
}

\begin{abstract}
We present a theory and Coulomb and Spin Blockade spectroscopy experiments on quantum Hall droplets with controlled electron numbers $(\mathrm{N} 1, \mathrm{~N} 2)$ in laterally coupled gated quantum dots. The theory is based on the configuration interaction method (CI) coupled with the unrestricted Hartree-Fock (URHF) basis. It allows us to calculate the magnetic field evolution of ground and excited states of coupled quantum dots with large electron numbers. The method is applied to the spin transitions in the $(5,5)$ droplet. Preliminary experimental results demonstrate the creation of the $(5,5)$ droplet and its Spin Blockade spectra.
\end{abstract}

Key words: Quantum Dots, Quantum Hall Effects, Nano-structures

The coupled lateral quantum dots form artificial molecules with each dot playing the role of an artificial atom[1,2,3,4]. In strong magnetic field electrons form quantum Hall droplet in each quantum dot. In a double dot one can couple the quantum Hall droplets in a controlled way, and at filling factor $\nu=2$ effectively reduce the many-electron-double dot system to a two-level molecule[3], as illustrated in the inset to Fig.1. The singlet-triplet spin transitions of a two-level molecule have potential application as quantum gates. $[5,6,7,8]$ Here we present a theory of spin transitions in many-electron double quantum dots in a magnetic field and preliminary results of Spin Blockade spectroscopy (SB) on double dots with controlled electron numbers $(\mathrm{N} 1, \mathrm{~N} 2)$ in dot 1 and $\operatorname{dot} 2$.

Our theory is based on effective mass envelop function to describe the confined electrons in quantum dots. We consider electron motion to be quasitwo-dimensional and coupled to the perpendicular external magnetic field by vector potential $A$. With the total number of electrons $N=N 1+N 2$ the quantum dot molecule Hamiltonian can be written as:

$H=\sum_{i=1}^{N} T_{i}+\frac{e^{2}}{2 \epsilon} \sum_{i \neq j} \frac{1}{\left|\mathbf{r}_{i}-\mathbf{r}_{j}\right|}$,

where $T=\frac{1}{2 m^{*}}\left(\frac{\hbar}{i} \nabla+\frac{e}{c} A(\mathbf{r})\right)^{2}+V(x, y)$ is the one electron Hamiltonian with $V(\mathbf{r})$ the quantum dot molecule confining potential, $m^{*}$ the conduction-electron effective mass, and $\epsilon$ the host semiconductor dielectric constant. The Zeeman spin splitting (very small for GaAs) is neglected here. In what follows we use GaAs effective atomic energy and length units with $R y^{*}=5.93 \mathrm{meV}$, and $a_{0}^{*}=9.79 \mathrm{~nm}$.

The double quantum dot potential $V(x, y)$ defined by electrostatic gates is characterized by two potential minima. With our focus on electronic correlations, we parameterize electrostatic 


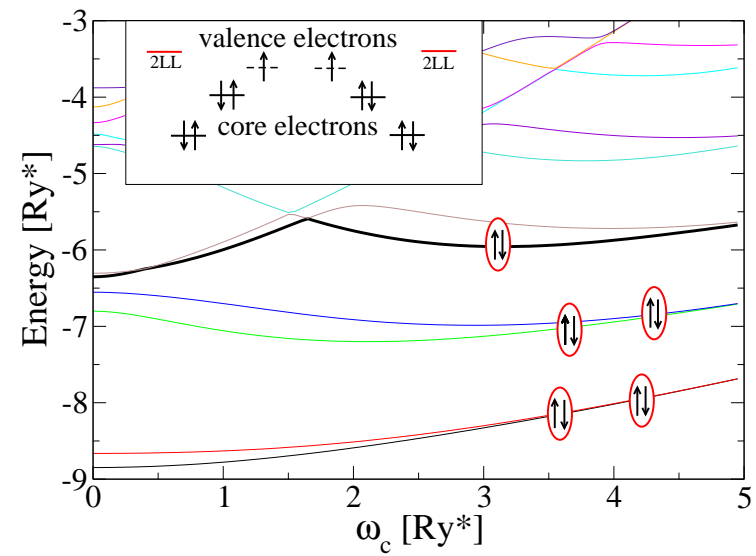

Fig. 1. Double dot single particle spectrum vs. cyclotron energy.

potential of a general class of coupled quantum dots by a sum of three Guassians [8] $V(x, y)=$ $V_{1} \quad \exp \left[-\frac{(x+a)^{2}+y^{2}}{\Delta^{2}}\right]+V_{2} \quad \exp \left[-\frac{(x-a)^{2}+y^{2}}{\Delta^{2}}\right]+$ $V_{p} \exp \left[-\frac{x^{2}}{\Delta_{P x}^{2}}-\frac{y^{2}}{\Delta_{P y}^{2}}\right]$. Here $V_{1}, V_{2}$ describe the depth of the left and righ quantum dot minima located at $x=-a, y=0$ and $x=+a, y=0$, and $V_{p}$ is the plunger gate potential controlled by the central gate. For identical dots, $V_{1}=V_{2}$, and confining potential exhibits inversion symmetry. In what follows we will parameterize it by $V_{0}=$ $-10, a=2, \Delta=2.5$, and $\Delta_{P x}=0.3, \Delta_{P y}=2.5$, in effective atomic units. $V_{p}$, which controls the potential barrier, is varied between zero and $10 R y^{*}$, independent of the locations of the quantum dots.

The potential of each isolated dot is a single Gaussian potential. Expanding it in the vicinity of the minimum yields a parabolic potential $V(r)=$ $1 /\left(2 m^{*}\right) \omega_{0} r^{2}$ with the strength $\omega_{0}=2 \sqrt{\left|V_{0}\right| / \Delta^{2}}$. The low energy spectrum of each dot corresponds to two harmonic oscillators with eigen-energies $\varepsilon_{n m}=\hbar \omega_{+}(n+1 / 2)+\hbar \omega_{-}(m+1 / 2)$. Here $\omega_{ \pm}=\sqrt{\omega_{0}^{2}+\omega_{c}^{2} / 4} \pm \omega_{c} / 2, \omega_{c}$ is the cyclotron energy, and $n, m=0,1,2, \ldots$. With increasing magnetic field the $\hbar \omega_{-}$decreases to zero while $\hbar \omega_{+}$approaches the cyclotron energy $\omega_{c}$, and the states $|m, n\rangle$ evolve into the nth Landau level. The $\nu=2$ spin singlet quantum Hall droplet is formed if $2 \mathrm{~N}$ electrons occupy the $\mathrm{N}$ successive $|m, n=0\rangle$ lowest Landau level (LLL) orbitals. When extra $2 N+1$ th electron is added it occupies the edge orbital $|m=N, n=0\rangle$. These $(2 \times 2+1,2 \times 2+1)$ configurations, for the two isolated dots, are shown as inset in Fig.1. In each isolated dot increasing the magnetic field leads to spin flips while decreasing the magnetic field lowers the energy of the edge $|m=N, n=0\rangle$ orbital with respect to the lowest unoccupied center orbital $|m=0, n=1\rangle$ of the second Landau level (2LL). At a critical magnetic field a LL crossing occurs and the electron transfers from the edge orbital to central orbital, leading to redistribution of electrons from the edge to center.[9]

We now turn to the description of coupled dots in strong magnetic field $(n=0)$. For weak coupling we expect the $|m ; 1\rangle$ orbitals of the first dot to be coupled with the $|m ; 2\rangle$ orbitals of the second dot, and form the symmetric and anti-symmetric orbitals $|m ; \pm\rangle=|m ; 1\rangle \pm|m ; 2\rangle$. Therefore in high magnetic field we expect the formation of shells of closely spaced pairs of levels. This is illustrated in Fig. 1 which shows the magnetic field evolution of the numerically calculated single particle spectrum of a double dot. The spectrum is calculated accurately by discretizing in real space the single particle Hamiltonian $T$ and applying special gauge transformation. The resulting large matrices are diagonalized using conjugate gradient algorithms.

At zero magnetic field Fig. 1 shows the formation of hybridized S, P, and D shells. In high magnetic field the pairs of closely spaced levels $|m, \pm\rangle$ separated by $\approx \omega_{+}$are clearly visible.

We can now populate the $|m, \pm\rangle$ electronic shells and form states equivalent to $\nu=2$ droplets in coupled quantum dots. The half-filled shells correspond to $(N 1, N 2)=(1,1),(3,3),(5,5),(7,7)$.. while filled shells correspond to $(N 1, N 2)=$ $(2,2),(4,4),(6,6),(8,8)$.. configurations. The population of the $(5,5)$ configuration is shown in Fig.1. We expect the half and fully filled shells to have special electronic properties. In particular, the half filled shells offer the possibility of singlet-triplet transitions. For the shells $(5,5)$ and up we expect to be able to move the valence electrons from the edge orbitals to the center orbitals. The crossing 




Fig. 2. URHFA ground state energy vs. cyclotron energy is shown for a quantum dot molecule. Spin transitions from $S=1$ to $S=3$, and from $S=3$ to $S=5$ occur at $\hbar \omega_{c}=3.4 R y^{*}$, and $\hbar \omega_{c}=4.9 R y^{*}$ (inset).

of the edge and center orbitals is visible as a cusp in the energy of the fifth molecular orbital shown as a bold line in Fig.1.

We now proceed to include electron-electron interactions in two steps: direct and exchange interaction using unrestricted Hartree-Fock approximation (URHF), and correlations using URHF basis in the configuration interaction method (URHF-CI). The spin-dependent HF orbitals $\left|\varphi_{i \sigma}\right\rangle$ are obtained from the $N_{l}$ non-interacting single particle orbitals $\left|\tilde{\varphi}_{\alpha}\right\rangle$, energy spectrum of which is shown in Fig.1, by the transformation $\left|\varphi_{i \sigma}\right\rangle=$ $\sum_{\alpha=1}^{N_{l}} a_{\alpha \sigma}^{(i)}\left|\tilde{\varphi}_{\alpha}\right\rangle$. The variational parameters $a_{\alpha \sigma}^{(i)}$ are solutions of self-consistent Pople-Nesbet equations [10]:

$$
\begin{aligned}
& \sum_{\gamma=1}^{N_{l}}\left\{\tilde{\epsilon}_{\mu} \delta_{\gamma \mu}+\sum_{\alpha, \beta=1}^{N_{l}} \tilde{V}_{\mu \alpha \beta \gamma}\left[\sum_{j=1}^{N_{\uparrow}} a_{\alpha \uparrow}^{*(j)} a_{\beta \uparrow}^{(j)}+\sum_{j=1}^{N_{\downarrow}}\right.\right. \\
& \left.\left.a_{\alpha \downarrow}^{*(j)} a_{\beta \downarrow}^{(j)}\right]-\tilde{V}_{\mu \alpha \gamma \beta} \sum_{j=1}^{N_{\uparrow}} a_{\alpha \uparrow}^{*(j)} a_{\beta \uparrow}^{(j)}\right\} a_{\gamma \uparrow}^{(i)}=\epsilon_{i \uparrow} a_{\mu \uparrow}^{(i)},
\end{aligned}
$$

where $\tilde{V}_{\alpha \beta \mu \nu}$ are Coulomb matrix elements calculated using non-interacting single particle states. A similar equation holds for spin down electrons. The calculations are carried out for all possible total spin $S_{z}$ configurations. The calculated total energies for the $N=10$ electrons with $S_{z}=0,1, . ., 5$ in a magnetic field are shown in Fig. 2. We find the lowest energy state to correspond to $S_{z}=1$ up to

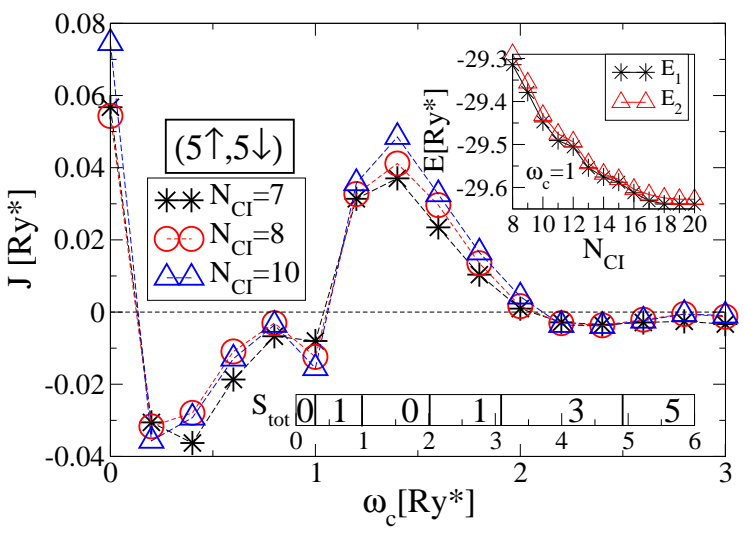

Fig. 3. The energy difference $\mathrm{J}$ between the triplet and the singlet ground states of the $(5,5)$ quantum Hall droplets from URHF-CI as a function of cyclotron energy $\omega_{c}$. The total spin $S_{\text {tot }}$ in magnetic field is shown in the lower inset. The ground state and first excited state energy as a function of $N_{S}$, the number of HF basis used in CI calculation, is shown in the upper inset.

$\hbar \omega_{c}=3.4 R y^{*}, S_{z}=3$ for $3.4 R y^{*}<\hbar \omega_{c}<4.9 R y^{*}$, and $S_{z}=5$ for $\hbar \omega_{c}>4.9 R y^{*}$. The predicted by URHFA total spin evolution with magnetic field is shown as inset in Fig. 2. It is remarkable to note that the $S=0$ singlet $(5,5)$ state is never a ground state, and the URHF picture is drastically different from the noninteraction picture presented in Fig.1. The situation is improved by including the electron correlation energy.

Correlations are included via CI method. Denoting the creation (annihilation) operators for URHF quasi-particles by $c_{i}^{\dagger}\left(c_{i}\right)$ with the index $i$ representing the combined spin-orbit quantum numbers, the many body Hamiltonian of the interacting system can be written as:

$H=\sum_{i j}\langle i|T| j\rangle c_{i}^{\dagger} c_{j}+\frac{1}{2} \sum_{i j k l} V_{i j k l} c_{i}^{\dagger} c_{j}^{\dagger} c_{k} c_{l}$,

where $\langle i|T| j\rangle=\epsilon_{i} \delta_{i j}-\left\langle i\left|V_{H}+V_{X}\right| j\right\rangle, V_{i j k l}$ are the Coulomb matrix elements in the URHF basis, $\epsilon_{i}$ are the URHF eigenenegies, $V_{H}$ and $V_{X}$ are the Hartree and exchange operators. The Hamiltonian matrix is constructed in the basis of configurations, and diagonalized using conjugated gradient methods for different total $S_{z}$. The convergence of CI calculation for the $(5,5)$ droplets has been checked by increasing the URHF basis up to $N_{S}=20$, associated with 240374016 configurations, with results shown as inset in Fig. 3. 

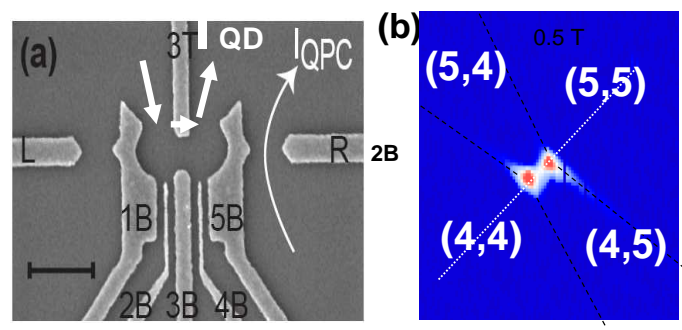

4B

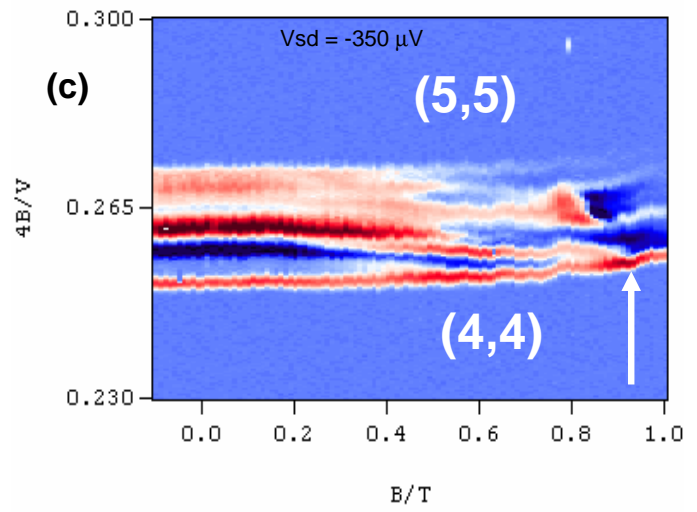

Fig. 4. (a) double dot gate layout, (b) transport through the ddot around $(5,5)$ configuration at $\mathrm{B}=0.5 \mathrm{~T}$, and (c) current stripe at finite source-drain voltage as a function of magnetic field.

The results of calculated exchange interaction $J \equiv E_{\text {triplet }}-E_{\text {singlet }}$, and the spin phase diagram of the $(5,5)$-droplet (inset) in magnetic field are shown in Fig. 3. Unlike in URHF, for magnetic fields less than $3.2 R y^{*}$, the ground state of the $(5,5)$-droplet oscillates between $S=0$ singlet state and $S=1$ triplet state. Correlations restored the $\nu=2$ quantum Hall droplet spin singlet $(S=0)$ phase as the phase within $1<\hbar \omega_{c}<2$. This phase is unstable against spin triplet state at $\hbar \omega_{c}=2$ and at $\hbar \omega_{c} \approx 1$. The spin singlet-triplet transition at $\hbar \omega_{c}=2$ is equivalent to the magnetic field induced singlet-triplet transition in a two-electron double $\operatorname{dot}[7]$. The spin singlet-triplet transition at $\hbar \omega_{c} \approx 1$ is associated with degeneracy of the crossing LLL edge and 2LL center orbitals.

At $\hbar \omega_{c}=3.2 R y^{*}$ spin state with $S=3$ is formed and at $\hbar \omega_{c}=4.8 R y^{*}$ a fully polarized state developes. The full spin evolution is summarized in the inset in Fig. 3, and will be discussed in detail in the future.

In order to test theoretical results we have designed a double dot with tunable electron numbers. The gate layout is shown in Fig.4a. Electrons are counted using both Coulomb and Spin Blockade spectroscopy and charge detection. Fig.4b shows current at $\mathrm{B}=0.5 \mathrm{~T}$ as a function of the $2 \mathrm{~B}$ and $4 \mathrm{~B}$ gates for device with $(5,5),(4,5),(5,4)$ and $(4,4)$ electron configurations. In Fig.4c we show the current stripe as a function of the magnetic field for a fixed source-drain voltage. The structures in the stripe correspond to excited states between the $(4,4)$ and $(5,5)$ configurations. The point indicated by the arrow suggests a transition in the double dot. We speculate that this transition corresponds to moving the 9th electron from the edge to the center orbital as shown in Fig.1. Much more work is needed to identify spin transitions in the $(5,5)$ droplet.

Acknowledgement. R.A. and P.H. acknowledge support by the NRC High Performance Computing project.

\section{References}

[1] J. J. Palacios and P. Hawrylak, Phys. Rev. B 51, 1769 (1995).

[2] W. G. van der Wiel et al., Rev. Mod. Phys. 75, 1 (2003).

[3] M. Pioro-Ladrire et al., Phys. Rev. Lett. 91, 026803 (2003).

[4] J.R. Petta et al, Phys. Rev. Lett. 93, 186802 (2004).

[5] J. A. Brum, and P. Hawrylak, Superlattices Microstruct. 22, 431 (1997);

[6] D. Loss and D. P. DiVincenzo, Phys. Rev. A 57, 120 (1998).

[7] G. Burkard, D. Loss and D. P. DiVincenzo, Phys. Rev. B 59, 2070 (1999).

[8] Xuedong $\mathrm{Hu}$ and S. Das Sarma, Phys. Rev. A 64, 042312 (2001).

[9] A.Wensauer, M.Korkusinski,P.Hawrylak, Phys.Rev.B 67, 035325, (2003).

[10] A. Szabo and N. S. Ostlund, Modern Quantum Chemistry (McGraw-Hill, New York, 1989). 